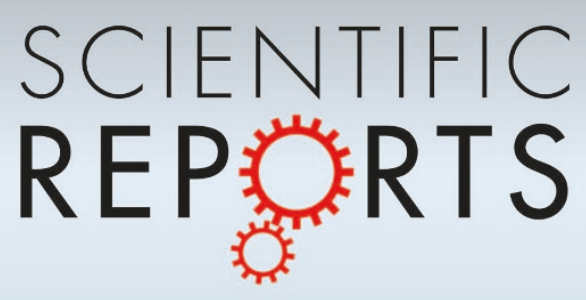

OPEN

SUBJECT AREAS:

TOPOLOGICAL

INSULATORS

SUPERCONDUCTING PROPERTIES

AND MATERIALS

COMPUTATIONAL METHODS

Received

24 October 2014

Accepted

10 February 2015

Published

10 March 2015

Correspondence and requests for materials should be addressed to W.-M.L. (wliu@iphy. ac.cn)

\section{Electron-Phonon Coupling and its implication for the superconducting topological insulators}

\author{
Xiao-Long Zhang \& Wu-Ming Liu
}

Beijing National Laboratory for Condensed Matter Physics, Institute of Physics, Chinese Academy of Sciences, Beijing 100190, China.

The recent observation of superconductivity in doped topological insulators has sparked a flurry of interest due to the prospect of realizing the long-sought topological superconductors. Yet the understanding of underlying pairing mechanism in these systems is far from complete. Here we investigate this problem by providing robust first-principles calculations of the role of electron-phonon coupling for the superconducting pairing in the prime candidate $\mathrm{Cu}_{x} \mathrm{Bi}_{2} \mathrm{Se}_{3}$. Our results show that electron-phonon scattering process in this system is dominated by zone center and boundary optical modes, with coexistence of phonon stiffening and softening. While the calculated electron-phonon coupling constant $\lambda$ suggests that $T_{c}$ from electron-phonon coupling is 2 orders smaller than the ones reported on bulk inhomogeneous samples, suggesting that superconductivity may not come from pure electron-phonon coupling. We discuss the possible enhancement of superconducting transition temperature by local inhomogeneity introduced by doping.

T he successful fabrication of topological insulators (TIs) ${ }^{1-4}$ has stimulated the search for other exotic topological matters. Among them the topological superconductors (TSCs) ${ }^{2}$ are of particular interest owing to its topologically protected gapless surface states consisting of massless Majorana fermions, which may find potential application in future topological quantum computation. The close relationship between TIs and $\mathrm{TSCs}^{2,5,6}$ indicates that topological superconductivity may born from TI. Indeed, there have been several attempts to induce topological superconductivity in $\mathrm{TI}^{7-12}$. In particular, the recent observation of superconductivity in doped topological insulators ${ }^{13-16}$ has received great attention and has been considered as prime candidates for TSCs.

The superconductivity in these doped topological insulators is unique since it occurs at relative low carrier concentration, and particularly the Dirac surface states remain intact with the onset of superconductivity in the case of $\mathrm{Cu}_{x} \mathrm{Bi}_{2} \mathrm{Se}_{3}{ }^{14}$. Shortly after the experimental discovery, $\mathrm{Fu}$ et al. ${ }^{17}$ argued that $\mathrm{Cu}_{x} \mathrm{Bi}_{2} \mathrm{Se}_{3}$ favors spin-triplet paring with odd-parity owing to its strong spin-orbit coupled band structure, and may realize a time-reversalinvariant (TRI) TSC. Although there is controversy on whether the Fermi surface of superconducting $\mathrm{Cu}_{x} \mathrm{Bi}_{2} \mathrm{Se}_{3}$ encloses odd number of TRI momenta ${ }^{18,19}$, which is key to a true $\mathrm{TSC}^{17}$, the prospect for a topologically nontrivial paring in this system is quite appealing. Indeed, subsequent point-contact spectroscopy measurements ${ }^{15}$ seem observe the zero bias conductivity peak which signifies the presence of Majorana surface states. But the result was challenged by other tunneling spectra measurements $\mathrm{s}^{20,21}$, leaving the topological aspect of superconductivity in this system has yet to be confirmed.

Despite much work concerning these systems to date ${ }^{22-28}$, the microscopic paring mechanism of the superconducting topological insulators remains elusive. On the one hand, unconventional superconductivity is generally associated with systems with strong electron correlations which seems unlikely for the typical topological insulators with sp electrons like $\mathrm{Bi}_{2} \mathrm{Se}_{3}$. On the other hand, whether electron-phonon (e-ph) coupling, which plays a central role in conventional BCS superconductivity, would realize the required unconventional paring is still an open question ${ }^{17,24,25}$. To address this problem, a comprehensive understanding of phonons and $e$-ph coupling in doped topological insulators is thus strongly called for.

In this Report, we present a systematic study of phonons and role of $e$-ph coupling for the representative system $\mathrm{Cu}_{x} \mathrm{Bi}_{2} \mathrm{Se}_{3}$ for a range of doping with accurate first-principles calculations. The e-ph properties are evaluated robustly by sampling the Brillouin zone (BZ) with several tens of thousands of inequivalent electron and phonon momenta. Our results reveal interesting renormalization of phonon dynamics due to electron doping, with coexistence of optical phonon stiffening and softening at zone center and boundary, signifying that the whole 

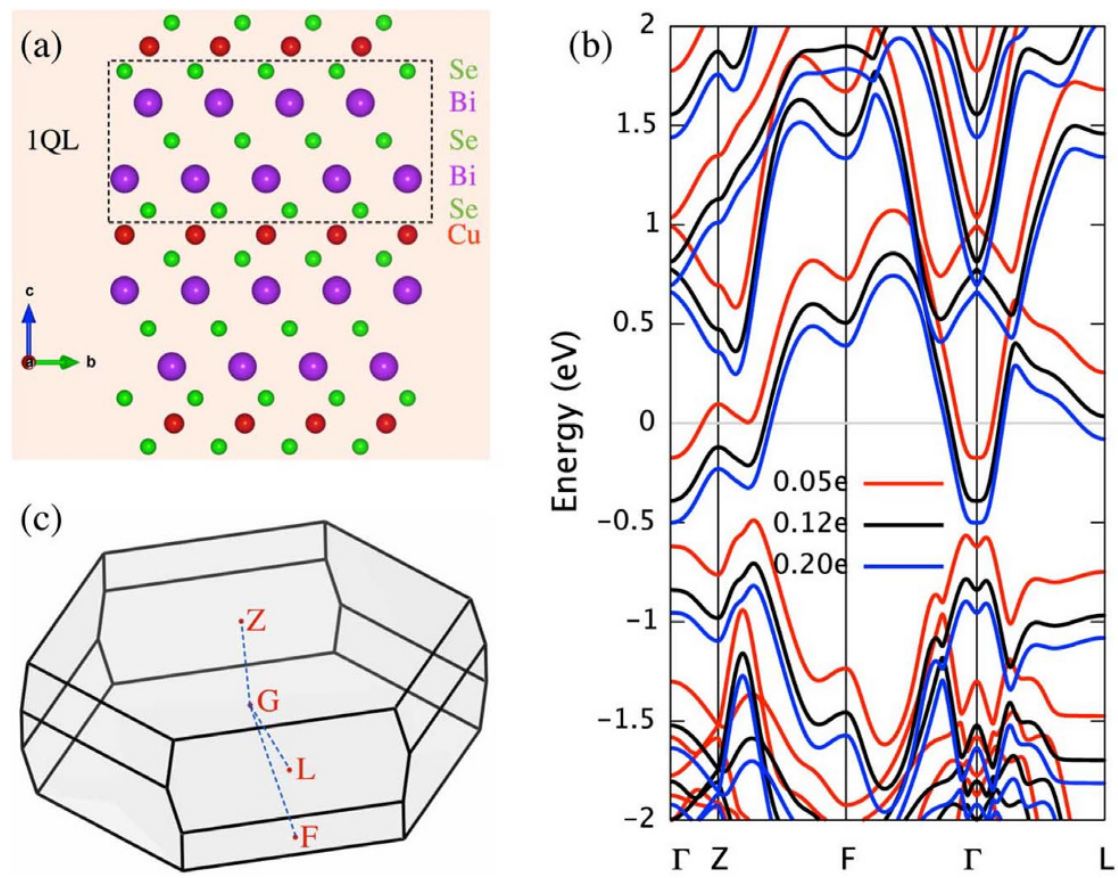

Figure $1 \mid$ The crystal structure of $\mathrm{Cu}_{\mathrm{x}} \mathrm{Bi}_{2} \mathrm{Se}_{3}$ and energy band evolution with electron doping. The crystal structure (a) and Brillouin zone (c) of $\mathrm{Cu}_{x} \mathrm{Bi}_{2} \mathrm{Se}_{3}$. (b) the electronic energy bands for 3 representative $\mathrm{Cu}$ doping $(x=0.05 e$ for an ellipsoidal Fermi surface, $x=0.12 e$ for a nearly cylindrical Fermi surface and $x=0.2 e$ corresponding to even more complex Fermi surface topology). To highlight the rigid shift of whole energy bands with electron doping, the Fermi level of each case has been set to 0 .

e-ph scattering process is dominated by these modes. Nevertheless, the obtained $e$-ph coupling constant $\lambda$ is 0.28 for the optimally doped $(x=0.12) \mathrm{Bi}_{2} \mathrm{Se}_{3}$, resulting in a maximum $T_{c}$ of only $\sim 0.03 \mathrm{~K}$, which is significantly smaller than the ones reported on inhomogeneous bulk ${ }^{13-16}$ but in line with very recent experiment where disorder and inhomogeneity are strongly suppressed ${ }^{29}$. Our results thus indicate that the superconductivity in $\mathrm{Cu}_{x} \mathrm{Bi}_{2} \mathrm{Se}_{3}$ may not be explained by only resorting to $e$-ph mechanism. We discuss the possibility of enhancement of superconducting transition temperature by local inhomogeneity introduced by copper doping.

\section{Results}

Doping induced strong renormalization of phonons dynamics. The $\mathrm{Bi}_{2} \mathrm{Se}_{3}$ crystallize in a rhombohedral structure with stacked Se$\mathrm{Bi}-\mathrm{Se}-\mathrm{Bi}$-Se quintuple layer (QL) as building blocks. Experimental data have consistently shown that copper atoms are mainly interpolated into the van der Waals gap between quintuple layers of $\mathrm{Bi}_{2} \mathrm{Se}_{3}$ and act as electron donor ${ }^{13,14,30}$. And the bulk electronic structures are virtually intact upon copper doping apart from a rigid shift of whole energy bands ${ }^{30,31}$ (see Fig. 1(b)). Hence we simulate doped topological insulator $\mathrm{Bi}_{2} \mathrm{Se}_{3}$ with the rigid-band approximation, where the excess of electron is compensated by uniform background of positive charge.

The vibrational properties of nominally undoped bulk $\mathrm{Bi}_{2} \mathrm{Se}_{3}$ have been extensively studied using Raman spectroscopy ${ }^{32,33}$ and all 4 Raman active optical phonon have been observed ${ }^{33}$. On the other hand, most available phonon data from fully-relativistic first-principles calculations are, however, unsatisfactory due to the presence of dynamical instability ${ }^{34,35}$. Our phonon dispersion does not suffer from this problem and the calculated zone center optical modes, which can be classified according to the irreducible representation of the point group of bulk $\mathrm{Bi}_{2} \mathrm{Se}_{3}$ as $\Gamma=2\left(E_{g}+A_{1 g}+E_{u}+A_{2 u}\right)$, agree well with experimental one ${ }^{33}$. The phonon spectrum extends up to $21.8 \mathrm{meV}$, and the vibrations of Se atoms mainly account for the top 9 optical branches and well separated in energy from Bi due to large mass difference.
When doped with copper atoms, the bulk conduction band of parent compound $\mathrm{Bi}_{2} \mathrm{Se}_{3}$ is partially filled due to charge transfer, and eventually develops superconductivity with copper concentration exceeds 0.10 when cooled down. Fig. 2 shows the phonon dispersions of bulk $\mathrm{Cu}_{x} \mathrm{Bi}_{2} \mathrm{Se}_{3}$ corresponding to several doping levels ( 0 $\leq \mathrm{x} \leq 0.14$ ) from a fully-relativistic calculation. As can be seen, while leaving the electronic structure almost unchanged ${ }^{30}$, electron doping strongly alters the vibrational properties of doped system, especially as reflected in the pronounced softening of the highest $A_{2 u}$ zone boundary modes ( $\mathrm{Z}$ point) and the coexistence of modes stiffening $\left(E_{u}\right)$ and softening $\left(A_{1 g}\right.$ and $\left.A_{2 u}\right)$ around zone center ( $\Gamma$ point). This doping-dependent phonon renormalization is most effect when doping starts $(x=0.05)$ and quickly saturates as the doping continues (especially at $Z$ point as can be seen from Fig. 2), reflecting different phonon dynamics of bulk insulating $\mathrm{Bi}_{2} \mathrm{Se}_{3}$ and electron-doped one due to electron screening. We note that in most experiments, the bulk sample of $\mathrm{Bi}_{2} \mathrm{Se}_{3}$ is usually slightly electron-doped owing to the presence of Se vacancies. Hence the abrupt change of phonon frequencies ( $\mathrm{Z}$ point) may not be easily observed. Indeed, previous Raman spectroscopy measurement ${ }^{36}$ showed no sign of significant change of phonon frequencies around zone center with $\mathrm{Cu}$ doping. Nevertheless, the special phonon stiffening and softening with electron doping here signifies these modes would dominate the $e$-ph scattering process. Actually, according to previous first-principles calculations $^{3}$, the energy bands around Fermi level are dominated by the hybridized $p_{z}$ orbitals from outermost Se atoms in a unit cell. And the $A_{2 u}$ mode at $\mathrm{Z}$ and $\Gamma$ involves out-of-phase motions of $\mathrm{Bi}$ and Se atoms along the $\mathrm{z}$-direction. Hence with electron doping, the partially occupied anti-bonding states would strongly interact with this phonon pattern, resulting in relatively large phonon linewidths as will be shown below.

Phonon linewidths from electron-phonon couping. The electron doping induced stiffening and softening of specific phonon modes is a clear sign of moderate $e$-ph interaction in this system. This is manifested in the relatively large phonon linewidths around zone 


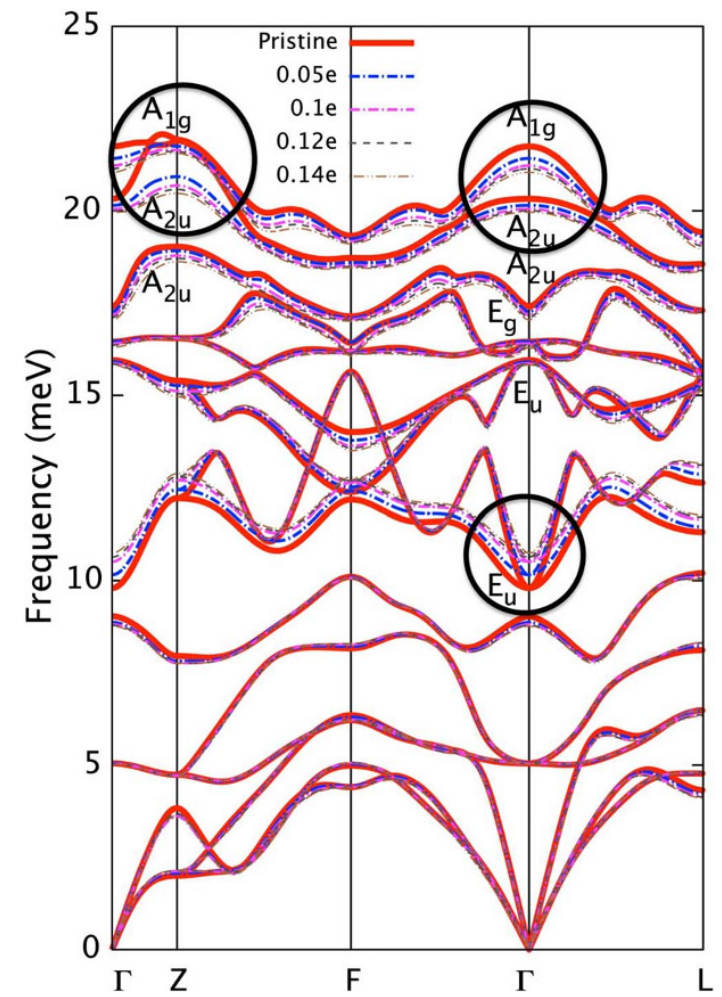

Figure $2 \mid$ The electron doping dependence of phonon dispersion. Phonon modes of interest are labeled according to the irreducible representation of point group of bulk $\mathrm{Bi}_{2} \mathrm{Se}_{3}$. The black circles highlight the major modifications of phonon modes $\left(A_{2 u}\right.$ at $\mathrm{Z;} A_{1 g}, A_{2 u}$ and $E_{u}$ at $\left.\Gamma\right)$ with copper doping.

center and zone boundary for the optimally doped $(x=0.12)$ $\mathrm{Cu}_{x} \mathrm{Bi}_{2} \mathrm{Se}_{3}$ (see Fig. 3). In calculating phonon linewidths, we have sampled the $\mathrm{BZ}$ with $50 \times 50 \times 50$ inequivalent electron wave vectors and the $\delta$ in Eq. (4) is replaced with 0.001 Ryd. From Fig. 3 we can see the significant phonon linewidths mainly lie in the top 9 optical branches which involve in-plane Se phonons and restricted to certain modes around the zone center $(\Gamma)$ and zone boundary $(Z)$ where phonon stiffening and softening occur. The phonon linewidths at other regions where phonon momentum $q>2 k_{f}$ ( $k_{f}$ is the electronic Fermi momentum) are negligible. This localized distribution of phonon linewidths resembles that in $\mathrm{MgB}_{2}{ }^{37,38}$, where the $E_{2 g}$ in-plane phonons near zone center strongly couples with partially occupied $\sigma$-bonding states and results in the largest $e$-ph coupling. This may suggest that $\mathrm{Cu}_{x} \mathrm{Bi}_{2} \mathrm{Se}_{3}$ is a good $e$-ph superconductor, but this is unfortunately not the case as will be discussed later.

There is another question need to be answered, namely how phonon linewidths evolve with electron doping, since the topology of Fermi surface (FS) of $\mathrm{Cu}_{x} \mathrm{Bi}_{2} \mathrm{Se}_{3}$ undergoes significant change from being an 3-D ellipsoid to 2-D cylindrical one $e^{19,39}$. It turns out that the doping dependence of phonon linewidths is negligible although the scattering phase space is increasing monotonically.

Role of $\boldsymbol{e}$-ph couping for superconductivity. Now let us turn to the $e$-ph coupling contribution to the superconductivity in $\mathrm{Cu}_{x} \mathrm{Bi}_{2} \mathrm{Se}_{3}$. It has been shown the superconductivity of $\mathrm{Cu}_{x} \mathrm{Bi}_{2} \mathrm{Se}_{3}$ occurs for a wide range of doping level $(0.10<x<0.60)^{40}$, and the highest superconducting transition temperature of $3.8 \mathrm{~K}$ is achieved at $x=$ 0.12 . Within the framework of Migdal-Eliashberg theory ${ }^{41,42}$, the superconductivity arising from $e$-ph coupling is characterized by several quantities ${ }^{43,44}$, the most important ones are Eliashberg spectral function

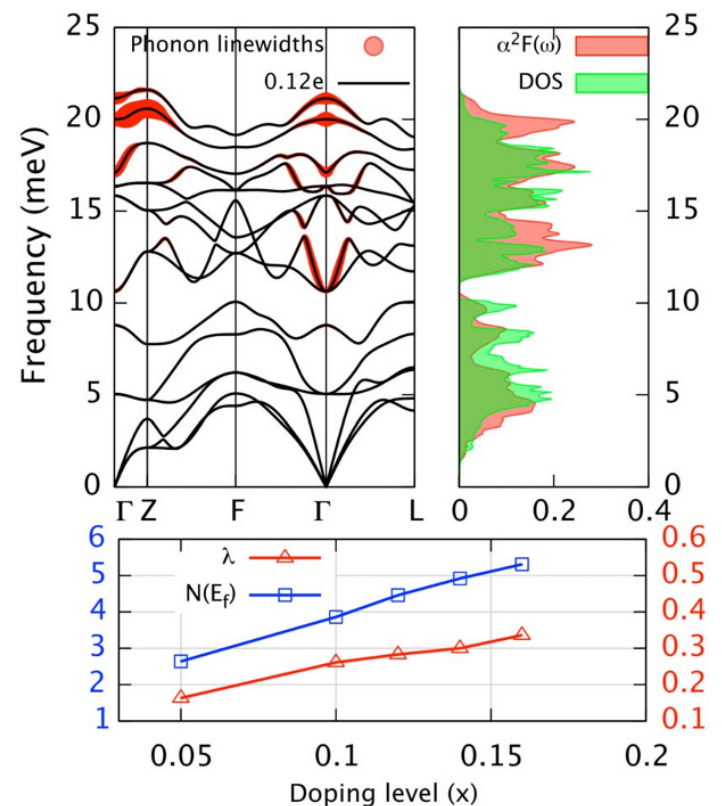

Figure $3 \mid$ The phonon linewidths for optimally doped $(x=0.12 \mathrm{e})$ $\mathrm{Cu}_{x} \mathrm{Bi}_{2} \mathrm{Se}_{3}$ and doping dependence of electron-phonon properties. Upper left: The radius of red circle is proportional to the magnitude of phonon linewidths. Upper right: Comparison of Eliashberg spectral function and phonon density of states for optimally doped case $(x=0.12 e)$. Lower: The doping dependence of electron-phonon coupling constant $\lambda$ (red point line) and electronic density of states at Fermi level $N\left(E_{f}\right)$ (blue point line).

$$
\alpha^{2} F(\omega)=\frac{1}{2} \sum_{v} \int_{B Z} \frac{d \mathbf{q}}{\Omega_{B Z}} \frac{1}{\pi N\left(E_{f}\right)} \frac{\gamma_{\mathbf{q} v}}{\omega_{\mathbf{q} v}} \delta\left(\omega-\omega_{\mathbf{q} v}\right),
$$

and the dimensionless $e$-ph coupling constant $\lambda=2 \int_{0}^{\infty} d \omega \omega^{-1}$ $\alpha^{2} F(\omega)$. As mentioned above, a reliable $\lambda$ needs dense sampling of both phonon and electron momentum. This is achieved here with the aid of Wannier interpolation, where the electronic and phonon states as well as $e$-ph coupling matrix elements are calculated on a fine mesh with $36 \times 36 \times 36$ and $30 \times 30 \times 30$ inequivalent electron and phonon momenta, respectively. This dense grid ensures the convergence of $\lambda$ to within 0.005 .

Fig. 3 summarizes our $e$-ph results for $\mathrm{Cu}_{x} \mathrm{Bi}_{2} \mathrm{Se}_{3}$. From the comparison of phonon DOS and Eliashberg spectral function we find that the $\alpha^{2} F(\omega)$ generally follow the trend of phonon DOS for the lowlying $\mathrm{Bi}$ modes $(\omega<10 \mathrm{meV})$ while deviate for the high-lying Se modes, and the discrepancy is significant around $13 \mathrm{meV}$ and $20 \mathrm{meV}$. Such behavior has been seen in $\mathrm{MgB}_{2}$, where the most significant contribution to the remarkable high $T_{c}$ of $39 \mathrm{~K}$ comes from phonon modes around $60 \mathrm{meV}^{38}$. Despite this resemblance, the $e$-ph coupling constant $\lambda$ for the optimally doped $(x=0.12)$ $\mathrm{Cu}_{x} \mathrm{Bi}_{2} \mathrm{Se}_{3}$ is 0.28 . The corresponding transition temperature $T_{c}$ can be obtained from Allen-Dynes formula ${ }^{43}$ :

$$
T_{c}=\frac{\omega_{\log }}{1.2} \exp \left[\frac{-1.04(1+\lambda)}{\lambda\left(1-0.62 \mu^{*}\right)-\mu^{*}}\right],
$$

where

$$
\omega_{\log }=\exp \left[\frac{2}{\lambda} \int d \omega \frac{\alpha^{2} \mathrm{~F}(\omega) \log \omega}{\omega}\right]
$$

is the logarithmically averaged phonon frequency. From Fig. 4 we can conclude that for typical retarded Coulomb repulsion $\mu^{*}$ of 0.10 , the estimated $T_{c}$ is only $0.03 \mathrm{~K}$, which is 2 orders smaller than experimental value. If we assume a constant $\omega_{\log }(\sim 11 \mathrm{meV})$, to match the 


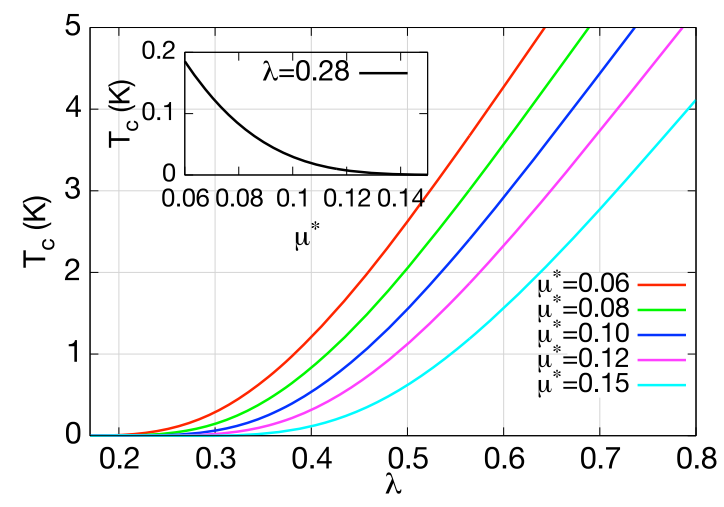

Figure $4 \mid$ The dependence of $T_{c}$ on $\lambda$ and $\mu^{*}$. $T_{c}$ has been plotted against $\lambda$ and $\mu^{*}$, from which we can estimate the lower bound of $\lambda$ to be $\sim 0.6$ to match the experimental $T_{c}$ of $4 \mathrm{~K}$. The inset indicates the calculated $T_{c}$ is far less than the experimental one for optimally doped case.

experimental value of $T_{c}$ the required $\lambda$ would be $0.6-0.7$ (with reasonable Coloumb $\mu^{*}$ of $0.06-0.12$, see Fig. 4 ), which is $\sim 2$ times larger than present one. Since the electronic DOS at Fermi level $N\left(E_{f}\right)$ is 4.46 states/Ryd/spin/(unit cell) which is almost identical to that in $\mathrm{MgB}_{2}$, it is clear that the major obstacle prevents $\mathrm{Cu}_{x} \mathrm{Bi}_{2} \mathrm{Se}_{3}$ from being a good e-ph superconductor as $\mathrm{MgB}_{2}$ (with $\lambda \sim 1$ ) is the lower characteristic phonon frequency $\left(\sim 60 \mathrm{meV}^{37}\right.$ for $\mathrm{MgB}_{2}$ but $\sim 11 \mathrm{meV}$ here) or Debye temperature $\left(\sim 900 \mathrm{~K}^{45}\right.$ for $\mathrm{MgB}_{2}$ but $\sim 180 \mathrm{~K}^{46}$ here).

In Fig. 3 we also plot the electron doping dependence of $\lambda$ and electronic DOS at Fermi level $N\left(E_{f}\right)$. At low doping $(x=0.05)$ where FS is a closed ellipsoid, the carrier concentration $(n)$ is $3.5 \times$ $10^{20} \mathrm{~cm}^{-3}$, slightly larger than the experimental one $(\sim 2.0 \times$ $\left.10^{20} \mathrm{~cm}^{-3}\right)^{13}$. The corresponding $N\left(E_{f}\right)$ is about 2.64 states/Ryd/ spin/(unit cell), and the calculated $\lambda$ for this doping is even smaller (being 0.16). When increasing electron doping to $x=0.10$, the $N\left(E_{f}\right)$ increased to $3.86 \mathrm{states} / \mathrm{Ryd} / \mathrm{spin} /($ unit cell), and the $\lambda$ jumps to 0.26 . Further increasing of doping will generally elevate $N\left(E_{f}\right)$ and $\lambda$, but the $\lambda$ is still unable to exceed 0.30 at $x=0.14$, although $n$ at this doping level is already one order larger than the experimental one, which is probably unrealistic since the ambipolar doping nature of $\mathrm{Cu}$ tends to make electron concentration saturate at $x=0.1^{30}$. Even higher doping would in addition result in an complex Fermi surface structure which is probably inconsistent with experiments ${ }^{19,39}$.

Thus, given the experimental facts (with $n \sim 2.0 \times 10^{20} \mathrm{~cm}^{-3}$ and an ellipsoidal or cylindrical FS), the value of 0.28 corresponding to optimal doping has been the upper bound for $\lambda$ and actually has overestimated it since the $n$ is $\sim 4$ times larger than experimental one.

\section{Discussion}

Our results are actually in line with recent experiments on epitaxial $\mathrm{Cu}_{x} \mathrm{Bi}_{2} \mathrm{Se}_{3}$ with thickness between 6 quintuple layers $(\mathrm{QL})$ to $13 \mathrm{QL}^{29}$, where superconducting transition is never observed down to $0.8 \mathrm{~K}$ even though the $n$ is already comparable with bulk one $\left(\sim 10^{20} \mathrm{~cm}^{-3}\right)$, suggesting that electron doping alone could not afford for the observed $T_{c}$ of $\sim 4 \mathrm{~K}$ and in particular, a weak $e$-ph contribution to the observed superconductivity. Moreover, previous angle-resolved photoemission spectroscopy measurements of $e$-ph coupling in $\mathrm{Bi}_{2} \mathrm{Se}_{3}$ also suggest a relatively small $\lambda$ of $0.25^{47}$ and $0.17^{48}$, which are very close to our first-principles results.

Therefore, we have to resort to other mechanisms besides $e$-ph coupling to recover the observed $T_{c}$ of $\sim 4 \mathrm{~K}$. Since electronic states of $\mathrm{Cu}_{x} \mathrm{Bi}_{2} \mathrm{Se}_{3}$ around Fermi level are dominated by $p$ character ${ }^{3}$, we would expect minor contribution from spin-fluctuations either. On the other side, we note that the $\mathrm{Cu}$-intercalated structure is formed both in bulk and films of $\mathrm{Cu}_{x} \mathrm{Bi}_{2} \mathrm{Se}_{3}{ }^{29,31}$, and the only difference is that disorder and inhomogeneity are strongly suppressed in latter case $\mathrm{e}^{29}$.
Indeed, there has been consistent observation of inhomogeneity of superconductivity in $\mathrm{Cu}_{x} \mathrm{Bi}_{2} \mathrm{Se}_{3}{ }^{13-16}$. And the superconducting shielding fraction reported also varies from group to group, in particular, Kriener et al. ${ }^{40}$ have shown that shielding fraction strongly depends on doping level $x$ and $T_{c}$ exhibits an unusual monotonic decrease with $x$, raising the possibility of phase segregation.

Hence we could infer that the local inhomogeneity introduced by copper may play nontrivial role in the superconductivity of $\mathrm{Cu}_{x} \mathrm{Bi}_{2} \mathrm{Se}_{3}{ }^{29,40}$. Indeed, the intimate relationship between local inhomogeneity and superconductivity has been extensively studied in the context of high $T_{c}$ superconductors, and the possible enhancement of superconductivity by local inhomogeneities has been discussed recently by Martin I. et al..$^{49}$ in the weak coupling BCS regime. Given many unusual properties of current system (relatively high $T_{c}$ compared to its low carrier density and the unexpected drop of shielding fraction for doping level $x>0.5)^{40}$, it is likely that the local inhomogeneity is indispensable to the superconductivity and may enhance $T_{c}$ of $\mathrm{Cu}_{x} \mathrm{Bi}_{2} \mathrm{Se}_{3}$.

Regarding the symmetry of the paring of superconducting $\mathrm{Cu}_{x} \mathrm{Bi}_{2} \mathrm{Se}_{3}$, no consensus has been reached since its first observation. Experimentally, point-contact spectroscopy measurements seem support the spin-triplet paring with odd-parity ${ }^{15}$, but results from scanning tunneling microscope and Andreev reflection spectroscopy show no evidence of characteristic zero-energy surface bound states $^{20,21}$, suggesting the picture of s-wave paring. On the other hand, based on a 2 band model, Fu et al. ${ }^{17}$ argued that $\mathrm{Cu}_{x} \mathrm{Bi}_{2} \mathrm{Se}_{3}$ favors spin-triplet paring owing to its strong spin-orbit coupled band structure, and the possibility of phonon-mediated odd-parity paring has also been discussed theoretically ${ }^{24,25}$. Our first-principles results here suggest the paring of this system may be unconventional, and this unconventionality may come from the local inhomogeneity introduced by copper doping. This is because the superconducting transition hasn't observed in epitaxial $\mathrm{Cu}_{x} \mathrm{Bi}_{2} \mathrm{Se}_{3}{ }^{29}$, which would rule out the possibility that superconductivity is mediated by phonon modes derived from pure $\mathrm{Bi}_{2} \mathrm{Se}_{3}$.

In summary, we have presented a systematic study of phonons and role of $e$-ph coupling in the $\mathrm{Cu}$ doped $\mathrm{Bi}_{2} \mathrm{Se}_{3}$. Our results show that strongly renormalized zone center and zone boundary modes with electron doping would dominate the whole $e$-ph coupling process. Despite moderate $e$-ph coupling in this system, our robust first-principles calculations of $e$-ph properties for wide range of copper doping suggest that $\mathrm{Cu}_{x} \mathrm{Bi}_{2} \mathrm{Se}_{3}$ is not a conventional $e$-ph superconductor and $e$-ph coupling plays minor role in superconductivity of this system. We have also discussed the possible enhancement of $T_{c}$ by the local inhomogeneity introduced by copper doping. Above all, our results rule out a conventional phonon-mediated superconductivity in $\mathrm{Cu}_{x} \mathrm{Bi}_{2} \mathrm{Se}_{3}$ and point to a delicate interplay between $e$-ph coupling of parent compound $\mathrm{Bi}_{2} \mathrm{Se}_{3}$ and nontrivial role played by inhomogeneity.

\section{Methods}

The $e$-ph properties have been obtained with the isotropic approximation to MigdalEliashberg theory ${ }^{41,42}$. In this framework, the phonon self-energy $\left(\Pi_{\mathbf{q} v}\right)$ arising from $e$ ph coupling is expressed as ${ }^{44}$

$$
\begin{aligned}
\Pi_{\mathbf{q} v}= & \sum_{m n} \int_{B Z} \frac{d \mathbf{k}}{\Omega_{B Z}}\left|g_{m n}^{v}(\mathbf{k}, \mathbf{q})\right|^{2} \times \\
& \frac{f_{n \mathbf{k}}-f_{m \mathbf{k}+\mathbf{q}}}{\epsilon_{n \mathbf{k}}-\epsilon_{m \mathbf{k}+\mathbf{q}}-\omega_{\mathbf{q} v}+i \delta},
\end{aligned}
$$

where $\epsilon_{n \mathbf{k}}$ is the electronic energy with band index $n$ and crystal momentum $\mathbf{k}$, and $\omega_{\mathbf{q} v}$ is the vibrational frequency with branch index $v$ and crystal momentum $\mathbf{q}$. The $f_{n \mathbf{k}}$ is the Fermi-Dirac distributions, and $\delta$ is a positive infinitesimal. The

$g_{m n}^{v}(\mathbf{k}, \mathbf{q})=\left\langle m \mathbf{k}+\mathbf{q}\left|\Delta v_{\mathbf{q} v}\right| n \mathbf{k}\right\rangle$ is the $e$-ph coupling vertex, where $\Delta V_{\mathbf{q} v}$ is the variation of the self-consistent potential induced by a collective ionic displacement. Note the spin degree has been incorporated into the band index.

The imaginary part of $\Pi_{\mathbf{q} v}$ corresponds directly to phonon half-width at halfmaximum $\gamma_{\mathbf{q} v}$, and the phonon mode-resolved $e$-ph coupling constant is given by 


$$
\lambda_{\mathbf{q} v}=\frac{1}{\pi N\left(E_{f}\right)} \frac{\gamma_{\mathbf{q} v}}{\omega_{\mathbf{q} v}^{2}},
$$

with $N\left(E_{f}\right)$ being the electronic density of states (DOS) at the Fermi level.

An accurate determination of phonon linewidths and hence $e$-ph coupling constant requires fine energy and momentum resolutions of electronic and phonon states as well as $e$-ph coupling matrix elements. We achieved this by the recently developed interpolation method through Maximally Localized Wannier Functions $(\mathrm{MLWFs})^{50,51}$, which has been demonstrated to be extremely successful in addressing $e$-ph properties p $^{52-55}$ and other electronic properties where ultra high density of momentum is needed ${ }^{56,57}$. As first step of this method, we employs standard density functional theory (DFT) ${ }^{58}$ and density functional perturbation theory (DFPT) ${ }^{59}$ to obtain converged ground state electronic density and dynamical matrix. In this study, we use experimental crystal structure with $a=4.138 \AA$ and $c=28.64 \AA$. Fully relativistic norm-conserving pseudopo-tentials is used for all the calculation here as relativistic corrections are necessary for a satisfactory quantitative description of topological insulator $\mathrm{Bi}_{2} \mathrm{Se}_{3}$. A kinetic energy cutoff of 35 Ryd with methfessel-paxton smearing widths of 0.01 Ryd and Monkhorst-Pack grids of $12 \times 12 \times 12$ for k point sampling are used to ensure the convergence of total energy. The dynamical matrix is obtained on a relatively coarse grid of $4 \times 4 \times 4$ phonon wave vectors (convergence has been achieved by comparing with phonon dispersion from $6 \times 6 \times 6 \mathrm{mesh}$ ).

Subsequently, the electronic and vibrational quantities obtained in first step are interpolated to dense grids which contains several tens of thousands of inequivalent phonon (electron) wave vectors. In this interpolation step, we first construct 30 spinor Wannier functions (using $p$-like atomic orbitals of $\mathrm{Bi}$ and $\mathrm{Se}$ ) to span a subset of full Hilbert space around Fermi level, on which the operators in momentum space like Hamiltonian and electron-phonon coupling matrix are projected. After obtaining operators in Wannier representation, we then interpolate back to the momentum space (Bloch representation) to obtain the converged results ${ }^{50,51}$. By doing this way, the $e$-ph coupling constant $\lambda$ is calculated on fine mesh grid containing $36 \times 36 \times 36$ and $30 \times 30 \times 30$ inequivalent electron and phonon momenta respectively. This dense grid ensures the convergence of $\lambda$ to within 0.005 .

1. Hasan, M. Z. \& Kane, C. L. Colloquium: Topological insulators. Rev. Mod. Phys. 82, 3045-3067 (2010).

2. Qi, X.-L. \& Zhang, S.-C. Topological insulators and superconductors. Rev. Mod. Phys. 83, 1057-1110 (2011).

3. Zhang, H. et al. Topological insulators in $\mathrm{Bi}_{2} \mathrm{Se}_{3}, \mathrm{Bi}_{2} \mathrm{Te}_{3}$ and $\mathrm{Sb}_{2} \mathrm{Te}_{3}$ with a single Dirac cone on the surface. Nat. Phys. 5, 438-442 (2009).

4. Chen, Y. L. et al. Experimental realization of a three-dimensional topological insulator, $\mathrm{Bi}_{2} \mathrm{Te}_{3}$. Science 325, 178-181 (2009).

5. Schnyder, A. P., Ryu, S., Furusaki, A. \& Ludwig, A. W. W. Classification of topological insulators and superconductors in three spatial dimensions. Phys. Rev. B 78, 195125 (2008).

6. Qi, X.-L., Hughes, T. L., Raghu, S. \& Zhang, S.-C. Time-reversal-invariant topological superconductors and superfluids in two and three dimensions. Phys. Rev. Lett. 102, 187001 (2009).

7. $\mathrm{Fu}, \mathrm{L} . \&$ Kane, C. L. Superconducting proximity effect and majorana fermions at the surface of a topological insulator. Phys. Rev. Lett. 100, 096407 (2008).

8. Linder, J., Tanaka, Y., Yokoyama, T., Sudbø, A. \& Nagaosa, N. Unconventional superconductivity on a topological insulator. Phys. Rev. Lett. 104, 067001 (2010).

9. Zhang, J. L. et al. Pressure-induced superconductivity in topological parent compound $\mathrm{Bi}_{2} \mathrm{Te}_{3}$. Proc. Natl. Acad. Sci. 108, 2428 (2011).

10. Wang, M.-X. et al. The coexistence of superconductivity and topological order in the $\mathrm{Bi}_{2} \mathrm{Se}_{3}$ thin films. Science 336, 5255 (2012).

11. Kirshenbaum, K. et al. Pressure-induced unconventional superconducting phase in the topological insulator $\mathrm{Bi}_{2} \mathrm{Se}_{3}$. Phys. Rev. Lett. 111, 087001 (2013).

12. Kong, P. P. et al. Superconductivity of the topological insulator $\mathrm{Bi}_{2} \mathrm{Se}_{3}$ at high pressure. J. Phys.: Condens. Matter. 25, 362204 (2013).

13. Hor, Y. S. et al. Superconductivity in $\mathrm{Cu}_{x} \mathrm{Bi}_{2} \mathrm{Se}_{3}$ and its implications for pairing in the undoped topological insulator. Phys. Rev. Lett. 104, 057001 (2010).

14. Wray, L. A. et al. Observation of topological order in a superconducting doped topological insulator. Nat. Phys. 6, 855-859 (2010).

15. Sasaki, S. et al. Topological superconductivity in $\mathrm{Cu}_{x} \mathrm{Bi}_{2} \mathrm{Se}_{3}$. Phys. Rev. Lett. 107, 217001 (2011).

16. Kriener, M., Segawa, K., Ren, Z., Sasaki, S. \& Ando, Y. Bulk superconducting phase with a full energy gap in the doped topological insulator $\mathrm{Cu}_{x} \mathrm{Bi}_{2} \mathrm{Se}_{3}$. Phys. Rev. Lett. 106, 127004 (2011).

17. Fu, L. \& Berg, E. Odd-parity topological superconductors: Theory and application to $\mathrm{Cu}_{x} \mathrm{Bi}_{2} \mathrm{Se}_{3}$. Phys. Rev. Lett. 105, 097001 (2010).

18. Lawson, B. J., Hor, Y. S. \& Li, L. Quantum oscillations in the topological superconductor candidate $\mathrm{Cu}_{0.25} \mathrm{Bi}_{2} \mathrm{Se}_{3}$. Phys. Rev. Lett. 109, 226406 (2012).

19. Lahoud, E. et al. Evolution of the fermi surface of a doped topological insulator with carrier concentration. Phys. Rev. B 88, 195107 (2013).

20. Levy, N. et al. Experimental evidence for s-wave pairing symmetry in superconducting $\mathrm{Cu}_{x} \mathrm{Bi}_{2} \mathrm{Se}_{3}$ single crystals using a scanning tunneling microscope. Phys. Rev. Lett. 110, 117001 (2013).

21. Peng, H., De, D., Lv, B., Wei, F. \& Chu, C.-W. Absence of zero-energy surface bound states in $\mathrm{Cu}_{x} \mathrm{Bi}_{2} \mathrm{Se}_{3}$ studied via andreev reflection spectroscopy. Phys. Rev. B 88, 024515 (2013).
22. Hao, L. \& Lee, T. K. Surface spectral function in the superconducting state of a topological insulator. Phys. Rev. B 83, 134516 (2011).

23. Nagai, Y., Nakamura, H. \& Machida, M. Rotational isotropy breaking as proof for spin-polarized cooper pairs in the topological superconductor $\mathrm{Cu}_{x} \mathrm{Bi}_{2} \mathrm{Se}_{3}$. Phys. Rev. B 86, 094507 (2012).

24. Wan, X. \& Savrasov, S. Y. Turing a band insulator into an exotic superconductor. Nat. Commun. 5, 4144 (2014).

25. Brydon, P. M. R., Sarma, S. D., Hui, H.-Y. \& Sau, J. D. Odd-parity superconductivity from phonon-mediated pairing. arXiv:1402.7061 (2014).

26. Hao, L. et al. Anisotropic spin-singlet pairings in $\mathrm{Cu}_{x} \mathrm{Bi}_{2} \mathrm{Se}_{3}$ and $\mathrm{Bi}_{2} \mathrm{Te}_{3}$. Phys. Rev. B 89, 214505 (2014).

27. Nagai, Y., Ota, Y. \& Machida, M. Nonmagnetic impurity effects in a threedimensional topological superconductor: From $p$ - to $s$-wave behaviors. Phys. Rev. B 89, 214506 (2014).

28. Mann, C. et al. Observation of coulomb repulsion between $\mathrm{Cu}$ intercalants in $\mathrm{Cu}_{x} \mathrm{Bi}_{2} \mathrm{Se}_{3}$. Phys. Rev. B 89, 155312 (2014).

29. Shirasawa, T. et al. Structure and transport properties of $\mathrm{Cu}$-doped $\mathrm{Bi}_{2} \mathrm{Se}_{3}$ films. Phys. Rev. B 89, 195311 (2014).

30. Tanaka, Y. et al. Evolution of electronic structure upon $\mathrm{Cu}$ doping in the topological insulator $\mathrm{Bi}_{2} \mathrm{Se}_{3}$. Phys. Rev. B 85, 125111 (2012).

31. Wang, Y.-L. et al. Structural defects and electronic properties of the $\mathrm{Cu}$-doped topological insulator $\mathrm{Bi}_{2} \mathrm{Se}_{3}$. Phys. Rev. B 84, 075335 (2011).

32. Richter, W. \& Becker, C. R. A raman and far-infrared investigation of phonons in the rhombohedral $\mathrm{V}_{2}-\mathrm{VI}_{3}$ compounds $\mathrm{Bi}_{2} \mathrm{Te}_{3}, \mathrm{Bi}_{2} \mathrm{Se}_{3}, \mathrm{Sb}_{2} \mathrm{Te}_{3}$ and $\mathrm{Bi}_{2}\left(\mathrm{Te}_{1-x} \mathrm{Se}_{x}\right)_{3}$ $(0<\mathrm{x}<1),\left(\mathrm{Bi}_{1-y} \mathrm{Sb}_{y}\right)_{2} \mathrm{Te}_{3}(0<\mathrm{y}<1)$. phys. stat. sol. (b) 84, 619-628 (1977).

33. Zhang, J. et al. Raman spectroscopy of few-quintuple layer topological insulator $\mathrm{Bi}_{2} \mathrm{Se}_{3}$ nanoplatelets. Nano Lett. 11, 2407-2414 (2011).

34. Cheng, W. \& Ren, S.-F. Phonons of single quintuple $\mathrm{Bi}_{2} \mathrm{Te}_{3}$ and $\mathrm{Bi}_{2} \mathrm{Se}_{3}$ films and bulk materials. Phys. Rev. B 83, 094301 (2011).

35. Wang, B.-T. \& Zhang, P. Phonon spectrum and bonding properties of $\mathrm{Bi}_{2} \mathrm{Se}_{3}$ : Role of strong spin-orbit interaction. Applied Physics Letters 100, 082109-082109-4 (2012).

36. Gnezdilov, V. et al. Helical fluctuations in the raman response of the topological insulator $\mathrm{Bi}_{2} \mathrm{Se}_{3}$. Phys. Rev. B 84, 195118 (2011).

37. Kong, Y., Dolgov, O. V., Jepsen, O. \& Andersen, O. K. Electron-phonon interaction in the normal and superconducting states of $\mathrm{MgB}_{2}$. Phys. Rev. B 64, 020501 (2001).

38. An, J. M. \& Pickett, W. E. Superconductivity of $\mathrm{MgB}_{2}$ : Covalent bonds driven metallic. Phys. Rev. Lett. 86, 4366-4369 (2001).

39. Lawson, B. J. et al. Quantum oscillations in $\mathrm{Cu}_{x} \mathrm{Bi}_{2} \mathrm{Se}_{3}$ in high magnetic fields. arXiv:1408.6852 (2014).

40. Kriener, M. et al. Electrochemical synthesis and superconducting phase diagram of $\mathrm{Cu}_{x} \mathrm{Bi}_{2} \mathrm{Se}_{3}$. Phys. Rev. B 84, 054513 (2011).

41. Migdal, A. B. Interaction between electrons and lattice vibrations in a normal metal. Sov. Phys. JETP 34(7), 996 (1958).

42. Eliashberg, G. M. Interactions between electrons and lattice vibrations in a superconductor. Sov. Phys. JETP 11, 696 (1960).

43. Allen, P. B. \& Dynes, R. C. Transition temperature of strong-coupled superconductors reanalyzed. Phys. Rev. B 12, 905-922 (1975).

44. Grimvall, G. The electron-phonon interaction in metals, vol. 16 (North-Holland Amsterdam, 1981).

45. Ravindran, P., Vajeeston, P., Vidya, R., Kjekshus, A. \& Fjellvåg, H. Detailed electronic structure studies on superconducting $\mathrm{MgB}_{2}$ and related compounds. Phys. Rev. B 64, 224509 (2001).

46. Shoemake, G. E., Rayne, J. A. \& Ure, R. W. Specific heat of n- and p-type $\mathrm{Bi}_{2} \mathrm{Te}_{3}$ from 1.4 to $90^{\circ}$ K. Phys. Rev. 185, 1046-1056 (1969).

47. Hatch, R. C. et al. Stability of the $\mathrm{Bi}_{2} \mathrm{Se}_{3}(111)$ topological state: Electron-phonon and electron-defect scattering. Phys. Rev. B 83, 241303 (2011).

48. Chen, C. et al. Tunable Dirac fermion dynamics in topological insulators. Sci. Rep. 3, 2411 (2013).

49. Martin, I., Podolsky, D. \& Kivelson, S. A. Enhancement of superconductivity by local inhomogeneities. Phys. Rev. B 72, 060502 (2005).

50. Giustino, F., Cohen, M. L. \& Louie, S. G. Electron-phonon interaction using Wannier functions. Phys. Rev. B 76, 165108 (2007).

51. Noffsinger, J. et al. EPW: A program for calculating the electronphonon coupling using maximally localized Wannier functions. Comput. Phys. Commun. 181, 2140-2148 (2010).

52. Choi, H. J., Roundy, D., Sun, H., Cohen, M. L. \& Louie, S. G. The origin of the anomalous superconducting properties of $\mathrm{MgB}_{2}$. Nature 418, 758-760 (2002).

53. Giustino, F., Yates, J. R., Souza, I., Cohen, M. L. \& Louie, S. G. Electron-phonon interaction via electronic and lattice Wannier functions: Superconductivity in boron-doped diamond reexamined. Phys. Rev. Lett. 98, 047005 (2007).

54. Park, C.-H., Giustino, F., Cohen, M. L. \& Louie, S. G. Electron- phonon interactions in graphene, bilayer graphene, and graphite. Nano letters $\mathbf{8}, 4229$ (2008).

55. Giustino, F., Cohen, M. L. \& Louie, S. G. Small phonon contribution to the photoemission kink in the copper oxide superconductors. Nature 452, 975 (2008).

56. Wang, X., Vanderbilt, D., Yates, J. R. \& Souza, I. Fermi-surface calculation of the anomalous hall conductivity. Phys. Rev. B 76, 195109 (2007).

57. Zhang, X.-L., Liu, L.-F. \& Liu, W.-M. Quantum anomalous hall effect and tunable topological states in 3d transition metals doped silicene. Sci. Rep. 3, 2908 (2013). 
58. Hohenberg, P. \& Kohn, W. Inhomogeneous electron gas. Phys. Rev. 136, B864-B871 (1964).

59. Baroni, S., de Gironcoli, S., Dal Corso, A. \& Giannozzi, P. Phonons and related crystal properties from density-functional perturbation theory. Rev. Mod. Phys. 73, 515-562 (2001).

60. Giannozzi, P. et al. Quantum espresso: a modular and open-source software project for quantum simulations of materials. J. Phys.: Condens. Matter. 21, 395502 (2009).

\section{Acknowledgments}

We would like to thank F. Giustino, X. T. Zhu, G. Q. Huang for fruitful discussion and the support from NKBRSFC under grants Nos. 2011CB921502, 2012CB821305, NSFC under grants Nos. 61227902, 61378017, SKLQOQOD under grants No. KF201403, SPRPCAS under grants No. XDB01020300. The first-principles calculation has been done with QUANTUM ESPRESSO code ${ }^{60}$, and electron-phonon interpolation has been done with modified EPW $\operatorname{code}^{51}$. The calculations were carried out at National Supercomputing Center in Tianjin of China.

\section{Author contributions}

X.L.Z. performed coding and calculations. X.L.Z., W.M.L. analyzed numerical results and contributed in completing the paper.

\section{Additional information}

Competing financial interests: The authors declare no competing financial interests.

How to cite this article: Zhang, X.-L. \& Liu, W.-M. Electron-Phonon Coupling and its implication for the superconducting topological insulators. Sci. Rep. 5, 8964; DOI:10.1038/ srep08964 (2015).

This work is licensed under a Creative Commons Attribution 4.0 International License. The images or other third party material in this article are included in the article's Creative Commons license, unless indicated otherwise in the credit line; if the material is not included under the Creative Commons license, users will need to obtain permission from the license holder in order to reproduce the material. To view a copy of this license, visit http://creativecommons.org/licenses/by/4.0/ 